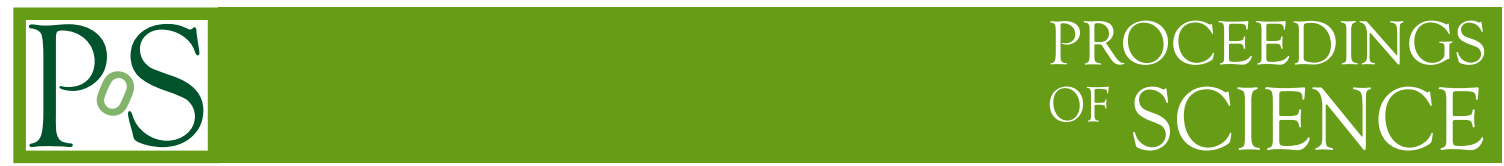

\title{
Lorentz completion of effective string action
}

\author{
Marco Meineri* \\ Scuola Normale Superiore, Piazza dei Cavalieri 7, 56126 Pisa, Italy \\ and Istituto Nazionale di Fisica Nucleare - sezione di Pisa \\ E-mail: marco.meineriesns.it
}

In the presence of a confining flux tube between a pair of sources the vacuum is no longer Poincaré invariant. This symmetry is nonlinearly realized in the effective string action. A general method for finding a large class of Lorentz invariant contributions to the action is described. The relationship between this symmetry and diffeomorphism invariance is further investigated.

Xth Quark Confinement and the Hadron Spectrum,

October 8-12, 2012

TUM Campus Garching, Munich, Germany

${ }^{*}$ Speaker. 


\section{Introduction}

The expectation value of large Wilson loops is an order parameter for the confining phase in non-abelian gauge theories. Indeed, a rectangular Wilson loop acts as a projector on the sector of the Hilbert space containing two non-dynamical sources, and the relative ground state energy of the sector w.r.t. the vacuum - i.e. the interquark potential - is easily extracted from the expectation value. As suggested by the phenomenon of Regge trajectories [1], a leading area law is expected to dominate the expectation value. Indeed, in this case the potential is linear in the separation, which indicates that a constant energy per unit length $\sigma$ is stored between the sources. The area law is in fact the leading term in a strong coupling expansion on the lattice, while higher order contributions organize themselves into a sum over surfaces with boundary on the loop. If one think of pushing the expansion all the way long to the continuum limit, one sees that the expectation value of large Wilson loops should be equal to the partition function of a two-dimensional field theory. This effective string theory describes the transverse fluctuations of a thin flux tube stretched between the sources. A first information on the action for the transverse coordinates comes again from the strong coupling expansion. Its radius of convergence does not extend until the continuum, because a roughening transition occurs [2], which is dominated by a Gaussian fixed point. The Euclidean action in the continuum should therefore look like

$$
\mathscr{S}_{\text {eff }}[X]=-\sigma R T-\frac{b_{0}}{2} \int_{0}^{T} d \xi^{0} \int_{0}^{R} d \xi^{1} \partial_{a} X^{i} \partial_{a} X^{i}+\ldots
$$

Here $\xi^{0}, \xi^{1}$ are the space-time coordinates on the plane where the Wilson loop lies, and latin indices from the beginning of the alphabet stand for them, while the fields $X^{i}\left(\xi^{a}\right)$ describe the embedding of the worldsheet. $T$ and $R$ are the temporal and spatial extensions of the rectangular loop. The explicit integration of the partition function leads to a universal attractive correction, the so called Lüscher term, so that in $D$ dimensions the potential becomes [3]

$$
V(R)=\sigma R-\frac{\pi(D-2)}{24 R}+\ldots
$$

The constant $b_{0}$ contributes to the width of the flux tube, which was measured for the first time in [4], and it turns out that the equality $b_{0}=\sigma$ holds. This equality is readily explained if one asks for a reparametrization invariant action. The Lüscher term has been unambiguously observed on the lattice $[5,6]^{1}$ and now numerical investigations can shed a light on what's behind the dots in (1.1). This low-energy action can be organized into a derivative expansion, and one has to rely on symmetries to fix the shape of the allowed terms. It has been recently realized $[9,10]$ that the Poincare symmetry of the underlying gauge theory provides a constructive method to tackle this problem $^{2}$. As already noticed, one should be free to choose the worldsheet parametrization, so that only a subgroup of the Poincare invariant expressions should appear in the action. However, it is not clear a priori if this subgroup coincides with the local functions of the metric induced by the embedding and of the second fundamental form, because the genuine degrees of freedom are just the transverse coordinates.

\footnotetext{
${ }^{1}$ see also [7], where the usual multilevel algorithm [8] is employed together with the tree-level improved action, and references therein.

${ }^{2}$ In the context of Galileon cosmology, the same perspective has been considered. See [11, 12, 13].
} 
In what follows, we shall briefly discuss how the effective action for a confining string is constrained by the spontaneous Poincare symmetry breaking due to the flux tube formation, and we shall then describe a systematic method for finding a large class of (in fact, possibly all) the Poincaré invariants [14]. In the end, the low-lying ones will be explicitly enumerated.

\section{Spontaneously broken Poincaré invariance}

Since the method we are meant to describe is independent of the number of dimensions and applies also to the general case of a $p$-brane, we shall keep our notation general, and we shall use Minkowski signature. The presence of a $d$-dimensional flat solitonic object in the ground state of a $D$-dimensional Poincaré invariant theory breaks spontaneously this symmetry down to the subgroup $I S O(1, d-1) \times S O(D-d)$. In particular, a Goldstone boson should appear for each of the broken translations, and the fields $X^{i}$ hold exactly this job. The symmetry allows them to appear in a low energy action. The roughening phenomenon is then understood in the continuum by means of the Mermin-Wagner-Coleman theorem [15]. Goldstone bosons undergo a nonlinear transformation under the broken generators $[16,17]$, but this reduces to a constant shift in the case of translations, which imposes that only field derivatives appear in the action. Since this is normally the case with Goldstone bosons anyhow, this makes little progress. However, the same fields are also the Goldstone bosons for the $d(D-d)$ broken Lorentz transformations (LT) [9]. Indeed, it is known that the number of massless modes no longer matches the number of broken generators, in the case of broken spacetime symmetries [18]. The nonlinear realization of a LT in the plane $(b, i)$ has been found as a linearly realized LT followed by a reparametrization, in the context of a gauge fixed diffeomorphism invariant theory [19, 9]:

$$
\delta_{\varepsilon}^{b i} X_{\mu}=\varepsilon\left(\delta_{\mu}^{b} X^{i}-\delta_{\mu}^{i} \xi^{b}-X^{i} \partial^{b} X_{\mu}\right)
$$

where a Greek index stands for a generic coordinate. However, it is not difficult to imagine at least an action which is invariant under the transformation (2.1) but not under reparametrization: a constant Lagrangian density provides this obvious example ${ }^{3}$. Therefore, this nonlinear transformation should be considered independently of the method used to derive it, and in the end its validity is ensured by the fact that it provides a realization of the algebra of LT. We have, for instance,

$$
\left[\delta_{\eta}^{c j}, \delta_{\varepsilon}^{b i}\right] X_{\mu}=-\varepsilon \eta\left[\delta^{i j}\left(\xi^{c} \delta_{\mu}^{b}-\xi^{b} \delta_{\mu}^{c}+\xi^{b} \partial^{c} X_{\mu}-\xi^{c} \partial^{b} X_{\mu}\right)+\eta^{b c}\left(X^{j} \delta_{\mu}^{i}-X^{i} \delta_{\mu}^{j}\right)\right] .
$$

In [10] the transformation rule (2.1) was systematically used for the first time to constrain the effective action. The interesting variations are the ones involving derivatives of the transverse fluctuations, which are easily seen to preserve the number of derivatives minus the number of fields. Following [10], we call this the scaling. Since terms of different scaling are unrelated by rotational symmetry, we can organize the invariants according to their scaling, at all orders in the derivative expansion. Moreover, since in the r.h.s. of (2.1) addends with a different number of derivatives are present, the requirement of a vanishing variation of the action will lead to recurrence relations for the couplings order by order. As we are going to show, it is not difficult to sum up the associated series in a large class of cases.

\footnotetext{
${ }^{3}$ Another example is provided by a tadpole, which could arise in the case of one transverse coordinate because $\delta X$ is a total derivative. However, we look for a homogeneous ground state in which $\langle X\rangle=0$, so we won't consider this possibility.
} 


\section{A diagrammatic method}

According to the symmetry breaking pattern, both worldvolume indices $(a, b, \ldots)$ and flavour indices $(i, j, \ldots)$ should be contracted. All polynomials in field derivatives obtained this way must be acted upon with the variation (2.1), and coefficients must be fixed by requiring the result to be a divergence. Let us begin by illustrating the procedure in the scaling zero case. It is useful to write the variation of the first derivative in a covariant form, $\varepsilon^{a j}$ being the matrix of parameters of the LT:

$$
\delta\left(\partial_{b} X^{i}\right)=-\varepsilon^{a j} \delta^{i j} \eta_{a b}-\varepsilon^{a j} \partial_{b} X^{j} \partial_{a} X^{i}-\varepsilon^{a j} X^{j} \partial_{a} \partial_{b} X^{i}
$$

Now we associate each expression which is invariant under the stability subgroup to a graph, where the nodes represent the field derivatives and are marked by the scaling (i.e. a node of the kind $d^{m} X^{n}$ is marked by $m-n$ ), and there are two kinds of link connecting the nodes. Solid lines represent saturation of worldvolume indices while wavy lines are associated to the saturation of transverse indices. At scaling zero the only possible structure is a disconnected set of polygons with an even number of vertices, i.e. a product of traces of powers of the matrix $\partial_{a} X^{i} \partial_{b} X^{i}$. One of these rings together with its variation is shown in fig.1. It is clear by inspection that by summing rings of increasing diameter with the appropriate coefficients the first two addends in the variation can be canceled. As for the third, it should be noticed that we get a total derivative by moving the solid link of the dot around from one vertex to the other. We therefore need to add to the Lagrangian density a copy of the first ring times a factor $(1 / 2) \partial_{a} X^{i} \partial^{a} X^{i}$ to complete the total derivative. This in turns is the beginning of a new ring, and we get another recurrence relation. It is easy to sum up the series and find the most general Lagrangian density at scaling zero ${ }^{4}$ up to an additive constant $-\sigma[14]$ :

$$
\mathscr{L}_{0}=-b_{0} \sqrt{-g}+b_{0}, \quad g=\operatorname{det} g_{a b}=\operatorname{det}\left(\eta_{a b}+\partial_{a} X^{i} \partial_{b} X^{i}\right) .
$$

If we choose $\sigma=b_{0}$ we obtain the diffeomorphism invariant Dirac-Born-Infeld action with vanishing electromagnetic field ${ }^{5}$.

When moving to higher scaling, higher derivatives enter the game. We call seeds the graphs which do not contain scaling zero nodes. The procedure we are going to set up permits to find

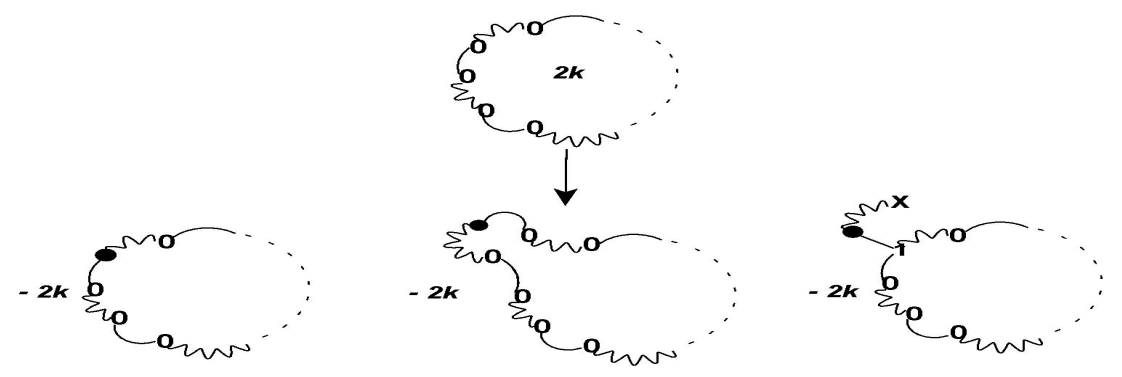

Figure 1: Variation of a ring at order $2 k$. The dot represents the matrix $\varepsilon^{a j}$ of parameters of the transformation, the 0 s and the $\mathbf{1}$ are first and second derivatives respectively, while the $\boldsymbol{X}$ is the non derived field.

\footnotetext{
${ }^{4}$ In fact, the Cayley-Hamilton theorem introduces linear dependences among rings of different sizes in every finite dimension. This leads to linear dependencies among invariants of the same scaling, but it's not difficult to argue that no new invariant can arise because of these relations.

${ }^{5}$ The case in which a photon propagates on the $p$-brane has been treated in [21]. See also [22].
} 
all invariants whose lowest order contribution is a linear combination of seeds. The variation of a field derivative of generic order can be expressed in a compact way by adopting the same notation explained for the graphs $(n>0)$ :

$$
\delta \boldsymbol{n}_{a_{1} \ldots a_{n+1}}^{i}=-\varepsilon^{a j} \sum_{k=-1}^{n} \sum_{\{p\}} \boldsymbol{k}_{a_{p(1)} \ldots a_{p(k+1)}}^{j}(\boldsymbol{n}-\boldsymbol{k})_{a a_{p(k+2) \ldots} \ldots a_{p(n+1)}}^{i}, \quad-\mathbf{1} \equiv X
$$

where the sum over $\{p\}$ counts the inequivalent ways of sharing the indices between $\boldsymbol{k}^{j}$ and $(\boldsymbol{n}-\boldsymbol{k})^{i}$. First of all, consider the $k=-1$ contribution. As in the scaling zero case, one has to add all the towers of growing rings in order to obtain a divergence. In other words, a Lorentz invariant of scaling $n>0$ has the form $\sqrt{-g} F_{n}$, where $F_{n}$ is a suitable (still infinite) linear combination of terms of scaling $n$. Then comes the contribution of $k=0$. The diagrammatic representation of this variation, together with the structure to be added to the Lagrangian density to enforce the symmetry (fig.2), shows that in this case too we get a recurrence relation. By summing the associated series one obtains the following rule: in each scalar product of the worldvolume indices, replace $\eta^{a b}$ with $g^{a b}$, where

$$
g^{a b}=\eta^{a b}-\eta^{a c} \partial_{c} X^{i} \partial_{d} X^{i} \eta^{d b}+\eta^{a c} \partial_{c} X^{i} \partial_{d} X^{i} \eta^{d e} \partial_{e} X^{j} \partial_{f} X^{j} \eta^{f b}-\ldots
$$

is the matrix inverse of $g_{a b}$. The contribution of $k=n$ can be treated in a similar manner. The rule in this case is the following: in each scalar product of the transverse coordinates, replace $\delta^{i j}$ with $t^{i j}$, where

$$
t^{i j}=\delta^{i j}-\partial_{a} X^{i} g^{a b} \partial_{b} X^{j}
$$

As for the other contributions in (3.3), which arise only for invariants of scaling four or higher, they correspond to all possible splittings of a node into two, whose scaling form a partition of the original one. As usual, in the terms to be added to the Lagrangian density a scaling zero node substitutes the dot (i.e. the matrix of coefficients of the transformation). It is easy to take into account the combinatorics in the diagrammatic representation, because the permutations in (3.3) simply correspond to all possible inequivalent ways of attaching the links to the new nodes. In fig.3 an example is shown. The generalization to higher order vertices is straightforward. It is maybe worth to notice that the bulk scalar products which involve scaling zero nodes, arising with this last procedure, should not be modified anymore. This completes the recipe to promote a seed, which is invariant under the action of the stability subgroup, to an expression which is invariant under the whole Lorentz group. In the next section we shall enumerate the invariants of lowest scaling.

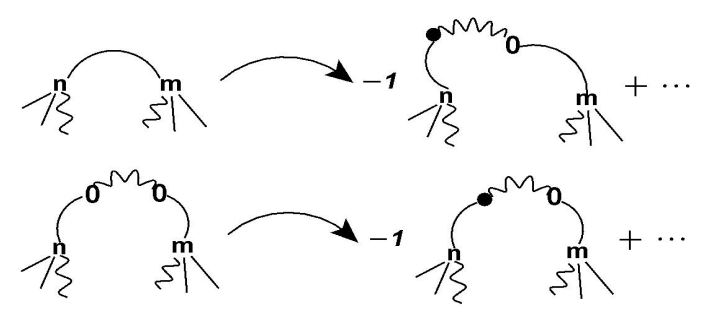

Figure 2: In the first line, a variation of the vertex $\boldsymbol{n}$. In the second line, the variation of the $\mathbf{0}$ on the left, which equals the first one. 


\section{The Lorentz invariant action}

At scaling zero, as already specified, the only Lorentz invariant Lagrangian densities are a constant and the volume form. In the case of the effective string, the explicit expression of the Nambu-Goto action is

$$
\mathscr{L}_{0}=-b_{0} \sqrt{-g}=-b_{0} \sqrt{1+\operatorname{Tr} h+\frac{1}{2}(\operatorname{Tr} h)^{2}-\frac{1}{2} \operatorname{Tr}\left(h^{2}\right)}, \quad h_{a b}=\partial_{a} X^{i} \partial_{b} X^{i} .
$$

At scaling two, there are two possible seeds, namely $\left(\partial_{a b}^{2} X \cdot \partial_{c d}^{2} X\right) \eta^{a c} \eta^{b d}$ and $\left(\partial_{a b}^{2} X \cdot \partial_{c d}^{2} X\right) \eta^{a b} \eta^{c d} \cdot{ }^{6}$ The associated invariants contain the two possible contractions of the squared second fundamental form $\left(K_{a b}^{i}\right):^{7}$

$$
\begin{aligned}
& \mathscr{L}_{2}^{1}=c_{0} \sqrt{-g}\left(\partial_{a b}^{2} X^{k} \partial_{c d}^{2} X^{k} g^{a c} g^{b d}-\partial_{a b}^{2} X^{k} \partial_{c d}^{2} X^{i} \partial_{e} X^{k} \partial_{f} X^{i} g^{a c} g^{b d} g^{e f}\right)=c_{0} \sqrt{-g} g^{a c} g^{b d} K_{a b}^{i} K_{c d}^{i} \\
& \mathscr{L}_{2}^{2}=d_{0} \sqrt{-g}\left(\partial_{a b}^{2} X^{k} \partial_{c d}^{2} X^{k} g^{a b} g^{c d}-\partial_{a b}^{2} X^{k} \partial_{c d}^{2} X^{i} \partial_{e} X^{k} \partial_{f} X^{i} g^{a b} g^{c d} g^{e f}\right)=d_{0} \sqrt{-g}\left(g^{a b} K_{a b}^{i}\right)^{2}
\end{aligned}
$$

Their difference is the Hilbert-Einstein action, which despite being a total derivative in the case of the effective string still plays a role in dimensional regularization [20]. It easy verified that the rules enforce the substitution $\partial_{a} \partial_{b} X^{i} \rightarrow \nabla_{a} \nabla_{b} X^{i}=K_{a b}^{j} n_{j}^{i}$, where $\nabla_{a}$ is the covariant derivative w.r.t. the induced metric and the vectors $n_{j}$ span the space normal to the brane in each point. Are the scaling four corrections still all diffeomorphism invariant? They are. In fact, the output of the three rules applied to the expression $\partial_{a} \partial_{b} \partial_{c} X^{i}$ is still covariant:

$$
\partial_{a} \partial_{b} \partial_{c} X^{i} \rightarrow \nabla_{a} \nabla_{b} \nabla_{c} X^{i}+\left(\nabla_{b} \nabla_{c} X^{j}\right)\left(\nabla_{a} \nabla_{d} X^{j}\right) g^{d e} \partial_{e} X^{i}=\left(\nabla_{a} K_{b c}^{j}\right) n_{j}^{i}
$$

Besides all scalars arising from traces of four copies of the second fundamental form, there is one more Lorentz invariant at scaling four, namely

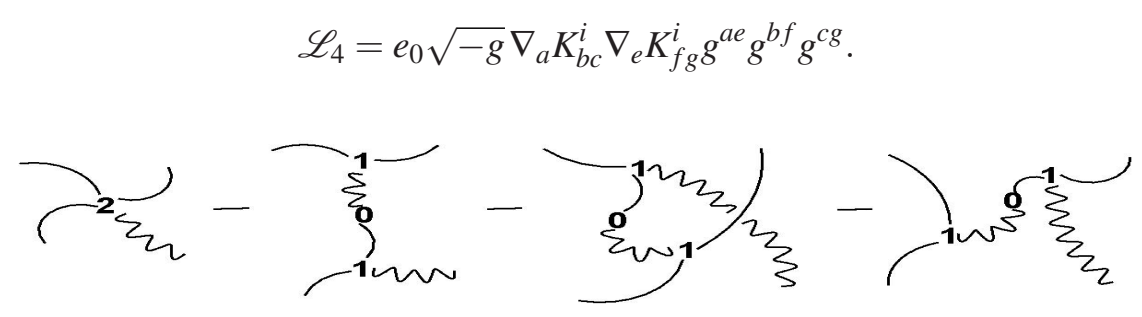

Figure 3: Splitting a vertex of scaling two.

\footnotetext{
${ }^{6}$ Notice that even if a seed is a total derivative up to the free equations of motion, and therefore it could be perturbatively swept out by field redefinitions, we take it into account. Indeed, the Lorentz invariant Lagrangian density we obtain by applying the rules established so far needs not to be a divergence up to the free e.o.m. (but it can be a divergence up to the DBI e.o.m.). When calculating the perturbative contribution to the interquark potential, these questions should be addressed.

${ }^{7}$ In the case of one transverse coordinate, it is easy to deduce from these expressions the existence of the invariant of scaling one $\mathscr{L}_{1} \propto \sqrt{-g} g^{a b} K_{a b}$, which cannot be directly detected in this general case (and is not parity invariant). However, it is easy to modify the procedure in order to work directly in this context.
} 


\section{Conclusions and perspectives}

The low energy effective action of a long confining flux tube has been studied under the only assumption of Lorentz invariance of the underlying gauge theory. From this perspective, the degrees of freedom we take into account are the Goldstone bosons of broken generators. Geometric quantities like the first and second fundamental forms are not introduced a priori, but arise as a consequence of the nonlinear realization of Lorentz algebra. When this approach was systematically introduced in [10], one of the main motivations was to set up a constructive way of detecting contributions to the effective action which are not local functions of geometric quantities, and could therefore escape a different analysis. We find no contributions of this kind up to scaling four apart from a constant, and it would be interesting to understand if this pattern extends to all orders. We leave this to future work. A first remark is that our analysis is completely classical: Lorentz algebra is plagued by anomalies at the quantum level, therefore new issues arise, and non-invariant counterterms have to be added to the action [20]. As for the constant, its presence in the action is irrelevant from a classical point of view. However, the interquark potential is determined by the entire partition function, therefore also this issue should be addressed from a quantum perspective [23]. When dealing with the effective string, boundary terms have to be considered. A general method to constrain the boundary action can be set up along the same guidelines illustrated here. In fact, the first correction compatible with Lorentz invariance comes from the boundary, and has been tested on the lattice $[24,25]$. It would be important also to isolate the signal of the first bulk correction to the Nambu-Goto action, which however is not allowed up to six derivatives [14, 26, 27].

\section{References}

[1] G.S. Bali, QCD forces and heavy quark bound states, Phys. Rept. 343 (2001), p.1 [hep-ph/0001312].

[2] C. Itzykson, M.E. Peskin, J. Zuber, Roughening transition of Wilson's surface, Phys. Lett. B95 (1980), p. 259.

[3] M. Lüscher, K. Symanzik, P. Weisz, Anomalies of the free loop wave equation in the WKB approximation, Nucl. Phys. B173 (1980), p.365.

[4] M. Caselle, F. Gliozzi, U. Magnea, S. Vinti, Width of long color flux tube in lattice gauge systems, Nucl. Phys. B460 (1996), p. 397 [hep-lat/9510019].

[5] M. Caselle, R. Fiore, F. Gliozzi, M. Hasenbusch, P. Provero, String effects in the Wilson loop: a high precision numerical test, Nucl. Phys. B486 (1997), p. 245 [hep-lat/9609041].

[6] M. Lüscher, P. Weisz, Quark confinement and the bosonic string, JHEP 0207 (2002), p. 049 [hep-lat/0207003].

[7] A. Mykkänen, The static quark potential from a multilevel algorithm for the improved gauge action, JHEP 1212 (2012) p. 069 [hep-lat/1209.2372].

[8] M. Lüscher, P. Weisz, Locality and exponential error reduction in numerical lattice gauge theory, JHEP 0109 (2001) p. 010 [hep-lat/0108014].

[9] O. Aharony, M. Field, On the effective theory of long open strings, JHEP 1101 (2011), p. 065 [hep-th/1008.2636]. 
[10] O. Aharony, M. Dodelson, Effective string theory and nonlinear Lorentz invariance, JHEP 1202 (2012) p. 008 [hep-th/1111.5758].

[11] A. Nicolis, R. Rattazzi, E. Trincherini, The Galileon as a local modification of gravity, Phys. Rev. D79 (2009) p. 064036 [hep-th/0811.2197].

[12] C. De Rham, A. Tolley, DBI and the Galileon reunited, JCAP 1005 (2010) p. 015 [hep-th/1003.5917].

[13] G. Goon, K. Hinterbichler, M. Trodden, Symmetries for Galileons and DBI scalars on curved space, JCAP 1107 (2011) p. 017 [hep-th/1103.5745].

[14] F. Gliozzi, M. Meineri, Lorentz completion of effective string (and p-brane) action, JHEP 1208 (2012) p. 056 [hep-th/1207.2912].

[15] M. Lüscher, Symmetry breaking aspects of the roughening transition in gauge theories, Nucl. Phys. B180 (1981) p. 317.

[16] S.R. Coleman, J. Wess, B. Zumino, Structure of phenomenological lagrangians I, Phys. Rev. 177 (1969) p. 2239.

[17] C.G. Callan, S.R. Coleman, J. Wess, B. Zumino, Structure of phenomenological lagrangians II, Phys. Rev. 177 (1969) p. 2247.

[18] I. Low, A.V. Manohar, Spontaneously broken space-time symmetries and Goldstone's theorem, Phys. Rev. Lett. 88 (2002), p. 101602 [hep-th/0110285].

[19] P. Goddard, J. Goldstone, C. Rebbi, C.B. Thorn, Quantum dynamics of a massless relativistic string, Nucl. Phys. B56, 109 (1973).

[20] S. Dubovsky, R. Flauger, V. Gorbenko, Effective string theory revisited, JHEP 1209 (2012) p. 044 [hep-th/1203.1054].

[21] F. Gliozzi, Dirac-Born-Infeld action from spontaneous breakdown of Lorentz symmetry in brane-world scenarios, Phys. Rev. D84 (2011), p. 027702 [hep-th/1103.5377].

[22] R. Casalbuoni, J. Gomis, K. Kamimura. Spacetime transformations of the Born-Infeld gauge field of a D-brane, Phys. Rev. D84 (2011), p. 027901 [hep-th/1104.4916].

[23] M. Lüscher, P. Weisz, String excitation energies in $S U(N)$ gauge theories beyond the free-string approximation, JHEP 0407 (2004) p. 014 [hep-th/0406205].

[24] M. Billò, M. Caselle, F. Gliozzi, M. Meineri, R. Pellegrini, The Lorentz-invariant boundary action of the confining string and its universal contribution to the inter-quark potential, JHEP 1205 (2012), p. 130 [hep-th/1202.1984].

[25] F. Gliozzi, The boundary action of the confining string and its universal contribution to the interquark potential, in proceedings of Xth Quark Confinement and the Hadron Spectrum.

[26] A. Athenodorou, B. Bringoltz, M. Teper, Closed flux tubes and their string description in D $=3+1$ $S U(N)$ gauge theories, JHEP 1102 (2011) p. 030 [hep-lat/1007.4720].

[27] A. Athenodorou, B. Bringoltz, M. Teper, Closed flux tubes and their string description in D=2+1 $S U(N)$ gauge theories, JHEP 1105 (2011) p. 042 [hep-lat/1103.5854]. 